\title{
Coexistence Challenges for Heterogeneous Cognitive Wireless Networks in TV White Spaces ${ }^{1}$
}

\author{
${ }^{1}$ C. Ghosh, ${ }^{1}$ S. Roy, and ${ }^{2}$ D. Cavalcanti \\ ${ }^{1}$ Electrical Engineering Department, University of Washington, Seattle, WA-98195 \\ ${ }^{2}$ Philips Research North America, Briarcliff Manor, NY \\ E-mail: ghoshc, sroy@u.washington.edu,dave.cavalcanti@philips.com
}

\begin{abstract}
In order to improve utilization of TV spectrum, regulatory bodies around the world have been developing rules to allow operation by secondary users in these bands provided that interference to incumbent broadcasters is avoided. Thus new services may opportunistically use temporarily unoccupied TV channels, known as Television White Spaces (TVWS). This has motivated several standardization efforts such as IEEE 802.22, 802.11af, 802.19 TG1, and ECMA 392 for furthering cognitive networking. Specifically, multiple co-located secondary networks are expected to use TVWS, each with distinct requirements (bandwidth, transmission power, and different system architecture and device types) that must all comply with regulatory requirements to protect incumbents. Heterogeneous coexistence in the TVWS is thus expected to be an important research challenge. This article introduces the current regulatory scenario, emerging standards for cognitive wireless networks targeting the TVWS, and discusses possible coexistence scenarios and associated challenges. Further, the article casts an eye on future considerations for these upcoming standards in support of spectrum sharing opportunities as a function of network architecture evolution.
\end{abstract}

Keywords: TV white space, heterogeneous cognitive wireless networks, spectrum sensing, interference mitigation, spectrum sharing

\section{INTRODUCTION}

The TV broadcasting spectrum is seen as one of the first opportunities to adopt and implement innovative and more efficient dynamic spectrum assess (DSA) models supported by cognitive radio technology [1]. With the transition to digital TV (e.g. June 2009 in the USA), considerable amount of vacant spectrum have been generated in the TV spectrum. This group of non-contiguous vacant channels is collectively known as $\boldsymbol{T V}$ White Spaces (TVWS) [2]. Regulatory efforts are currently ongoing in many countries to enable secondary access to TVWS, provided that harmful interference to incumbent services is avoided. Some examples include Federal Communications Commission (FCC) regulations [2] in the US, initiatives by OFCOM in UK, and the Electronic Communications Committee (ECC) in EU. The TVWS availability is time and location dependent and it may include the following portions of the radio spectrum: $54-72 \mathrm{MHz}, 76-88 \mathrm{MHz}, 174-216 \mathrm{MHz}$, and 470-806 $\mathrm{MHz}$.

The prospects of new spectrum availability subject to TVWS regulations have triggered development of new wireless standards. Some of the standardization activities targeting TVWS include IEEE 802.22 [3] for Wireless Regional Area Networks (WRAN), ECMA 392 [4] for personal/portable devices in TVWS, and most recently started IEEE 802.11af and 802.19.1 Task Groups. As a result, one can envision coexistence scenarios involving heterogeneous secondary systems and incumbents. An example is shown in Fig. 1 where a TV broadcasting station serves as an incumbent with receivers inside its protection contour. A low power wireless microphone is also an incumbent service,

\footnotetext{
${ }^{1}$ The work of C. Ghosh and S. Roy was supported in part by AFOSR Grant \# FA 9550-09-1-0298.
} 
although with a smaller protection foot-print as shown in Fig. 1. Secondary systems could include 802.22 WRANs [3] consisting of a Base Station (BS), fixed CPEs (Consumer Premise Equipments) and also mobile devices, as well as Wi-Fi home networks and hot spots operating in the TVWS. A number of scenarios involving low power devices for multimedia and Internet access in home and outdoor settings could also be envisioned.

Heterogeneity and coexistence are a characteristic of any unlicensed band and is not unique to TVWS. However, the dynamic nature of TVWS coupled with incumbent protection requirements poses new and subtle challenges that should be considered in achieving the end goals of improved spectrum utilization. The objective of this article is to provide an overview of heterogeneous coexistence issues in the TVWS and describe upcoming regulations and wireless standards that provide a framework to facilitate such coexistence. The challenges can be broadly classified into three categories: spectrum availability detection, interference mitigation and spectrum sharing. The outstanding issues encompass regulation requirements (e.g. spectrum sensing thresholds) and heterogeneities in operational characteristics of the secondary systems, including network architecture (e.g. master- slave, peer-to-peer, mesh, etc.), device category (fixed vs. personal/portable), transmission power limits, operational bandwidth, modulation/coding schemes, and medium access control (MAC) schemes (e.g. reservation or contention-based access). Further, we look at ideas proposed in wireless standards for TVWS in this regard and highlight open questions central to heterogeneous coexistence.

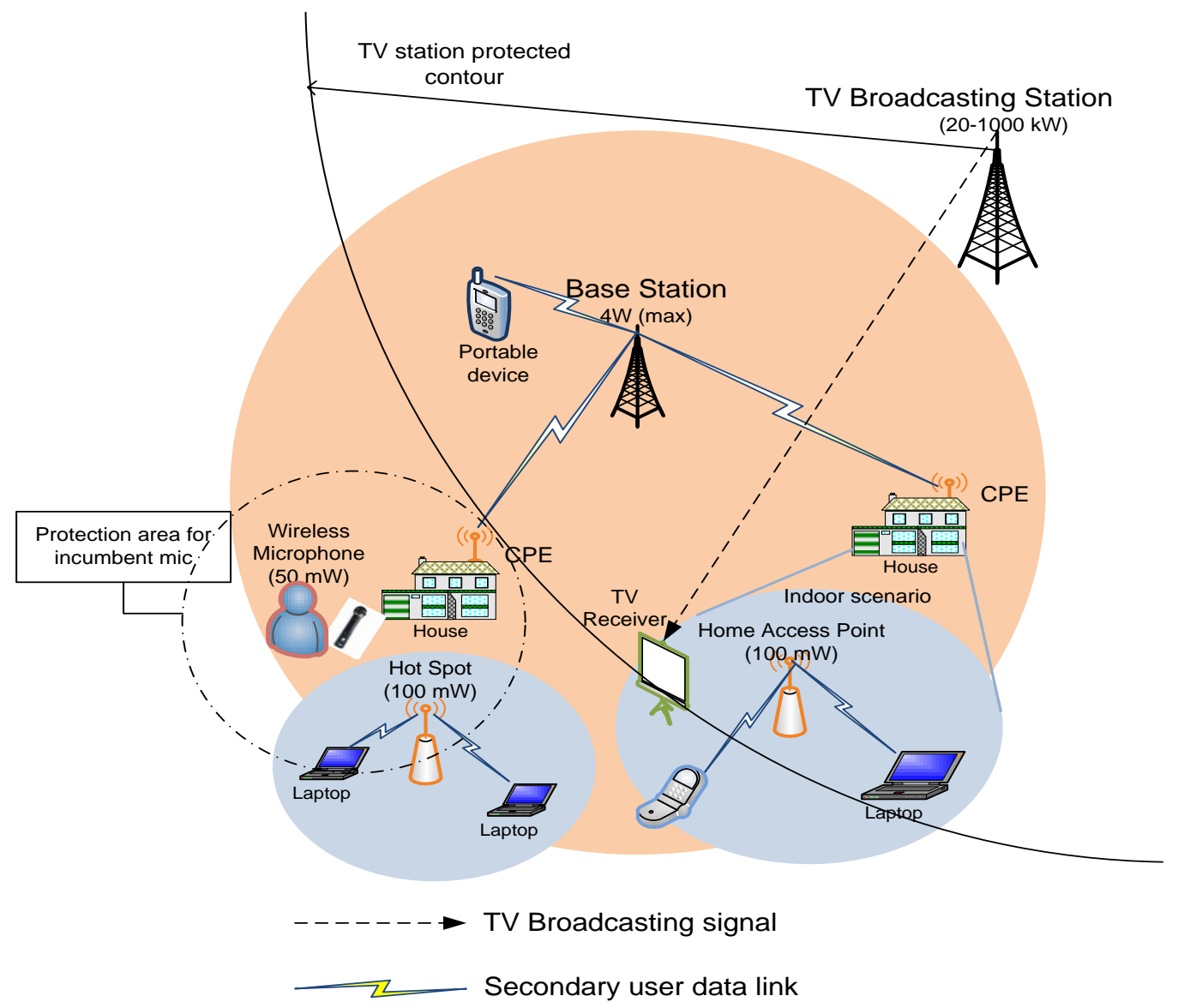

Fig. 1: A typical heterogeneous coexistence scenario with various networks and user applications with transmission power limits. 


\section{OVERVIEW OF REGULATION AND COGNITIVE RADIO (CR) STANDARDS}

\section{A. TVWS Regulations}

Global regulatory efforts to achieve more efficient spectrum utilization include the FCC regulations in the US and similar initiatives in Europe. The FCC published the first ground rules in $2008^{2}$ [1] that regulate secondary operation in TVWS (see Table I), where the devices are divided into two categories: fixed and personal/portable. Fixed devices can transmit up to $4 \mathrm{~W}$ EIRP (equivalent isotropically radiated power) and are required to have geolocation and spectrum sensing capability and a means to retrieve a list of available channels from an authorized database (see Sec. III-A). Personal/portable devices are allowed a maximum EIRP of $100 \mathrm{~mW}$ on non-adjacent channels and 40 $\mathrm{mW}$ on channels adjacent to an active TV broadcasting channel. Additional restrictions on channel operation are applicable to both fixed and portable device as shown in Table I. Devices are also classified into 2 modes: Mode I and Mode II. Mode II devices must possess geolocation, database access, and sensing capabilities while Mode I device only need sensing. The FCC rules also define sensing requirements: DTV and wireless microphone signals must be detected at a received signal level of $-114 \mathrm{dBm}$. A channel must be sensed for 30 seconds before determining if it is available for use. Once occupied, sensing must be done at least once every 60 seconds. If the incumbent is detected, the channel must be vacated within 2 seconds. These rules require adoption of cognitive radio techniques to deal with the fundamental challenges related to coexistence with incumbents as well as with other secondary systems, as discussed later in Section III.

\section{B. Standards for Cognitive Wireless Networks}

The IEEE 802.22 Working Group (WG) is developing a PHY and MAC layer specification for WRANs operation in TVWS. The primary application is fixed broadband access. The 802.22 standard (currently in its Draft 3.0) adopted an orthogonal frequency division multiple access (OFDMA) PHY and a centralized and connection-oriented MAC, where a BS controls resource allocations within its cell. The MAC layer is expected to provide user data rates of $1.5 \mathrm{Mbps}$ in the downlink and $384 \mathrm{Kbps}$ in the uplink and support up to $255 \mathrm{CPEs}$ per cell per $6 \mathrm{MHz}$ TV channel [3]. The 802.22 PHY and MAC layers include new CR features to protect incumbents and achieve efficient spectrum utilization such as reliable incumbent detection combining spectrum sensing, geolocation, and database; frequency agility; and self-coexistence mechanisms.

Table I: Overview of the FCC rules for TVWS

\begin{tabular}{|c|c|c|c|c|c|}
\hline \multicolumn{2}{|c|}{$\begin{array}{l}\text { Device types/ } \\
\text { Capability }\end{array}$} & $\begin{array}{l}\text { Allowed TV } \\
\text { Channels }\end{array}$ & Max EIRP & $\begin{array}{l}\text { Incumbent } \\
\text { protection } \\
\text { requirements }\end{array}$ & $\begin{array}{l}\text { Allowed on } \\
\text { Adjacent } \\
\text { channels }\end{array}$ \\
\hline \multicolumn{2}{|c|}{ Fixed } & $\begin{array}{l}\text { Ch } 2-51 \\
\text { (except Ch } \\
3,4 \text { and } 37 \text { ) }\end{array}$ & $4 \mathrm{~W}$ & $\begin{array}{l}\text { Geolocation/Database } \\
\text { Sensing }\end{array}$ & No \\
\hline \multirow{2}{*}{$\begin{array}{l}\text { Personal/ } \\
\text { Portable }\end{array}$} & $\begin{array}{l}\text { Mode I/ } \\
\text { Slave }\end{array}$ & \multirow{2}{*}{$\begin{array}{l}\text { Ch } 21-51 \\
\text { (except Ch } \\
37 \text { ) }\end{array}$} & $100 \mathrm{~mW}$ & Sensing & $\begin{array}{l}\text { Yes }(<40 \mathrm{~mW} \\
\text { EIRP) }\end{array}$ \\
\hline & $\begin{array}{l}\text { Mode II/ } \\
\text { Master }\end{array}$ & & $100 \mathrm{~mW}$ & $\begin{array}{l}\text { Geolocation/Database } \\
\text { Sensing }\end{array}$ & $\begin{array}{l}\text { Yes }(<40 \mathrm{~mW} \\
\text { EIRP) }\end{array}$ \\
\hline
\end{tabular}

\footnotetext{
${ }^{2}$ There have been several requests for reconsideration of the TVWS rules. The FCC is currently reviewing such request and is expected to publish a final position by the end of 2010. Therefore, changes to the rules are possible, although major changes to the core requirements are unlikely to happen.
} 
Different initiatives have also been proposed within the IEEE WGs 802.11, 802.22, and 802.19 targeting personal/portable device use cases that are considered to be future market drivers. The Task Group 802.11af (TGaf), approved in December 2009, is expected to define a new PHY and associated MAC layer modifications for TVWS operation. The 802.22 standard has also recently expanded its scope to allow portable CPEs to connect to the BSs, when they are in close proximity to the BS. However, the expanded 802.22 scope does not cover fully mobile CPEs at vehicular speeds. Unlike 802.22 and 802.11, 802.19 TG1 will not develop a new air-interface specification, but will focus on recommendations for broad coexistence protocols and policies across platforms for efficient spectrum utilization. Additionally, the recently released ECMA 392 standard [4] specifies PHY and MAC layers for operation in TVWS aimed at multimedia distribution and internet access for persona/portable devices. Other standardization efforts related to $\mathrm{CR}$ technology are ongoing in the IEEE SCC 41 group [5].

Other than ECMA 392 and 802.22, the 802.11af and 802.19 TG1 groups are in very early stages and specific technical solutions have not yet been proposed. However, some of the basic CR concepts, such as spectrum sensing and geolocation mechanisms, incumbent database access, and dynamic frequency selection will most likely be adapted to the specific requirements of each standard.

\section{HETEROGENEOUS COEXISTENCE CHALLENGES AND CONSIDERATIONS IN TVWS}

Once new standards and compatible products are developed, one can envision scenarios where multiple TVWS networks, hereafter referred to as Cognitive Wireless Networks (CWNs), will likely overlap with each other creating a need for coexistence mechanisms. A generic heterogeneous scenario is illustrated in Fig. 1, where multi-radio devices take advantage of the TVWS to achieve higher capacity and/or larger transmission ranges. In one typical case, a fixed broadband network (e.g. 802.22) could provide wireless backhaul to homes, which use Wi-Fi (e.g. 802.11af) or Ecma 392 for in-home coverage. Alternately, 802.11af or Ecma 392 devices could form a neighborhood mesh network.

Fig. 2 illustrates the main challenges in heterogeneous CWNs with implications for all layers of the protocol stack. The issues, typically related to PHY and MAC layers, can be grouped in three categories: spectrum availability detection, spectrum sharing, and interference mitigation; these are discussed next.

\section{A. Spectrum Availability Detection}

Spectrum availability detection refers to the problem of identifying TV channels available for use without causing harmful interference to incumbents. In addition, detection of coexisting secondary networks is also important, primarily to enable optimized decisions when selecting operating channels.

\section{Incumbent detection}

The CWNs must apply reliable methods to detect available TVWS. For instance, the FCC (see Table I) requires secondary systems to determine an available TV channel using two methods:

- White space database (WSD) access: A WSD is a central repository managed by a secure and reliable authority. It stores information about primary user operations (i.e., location of 


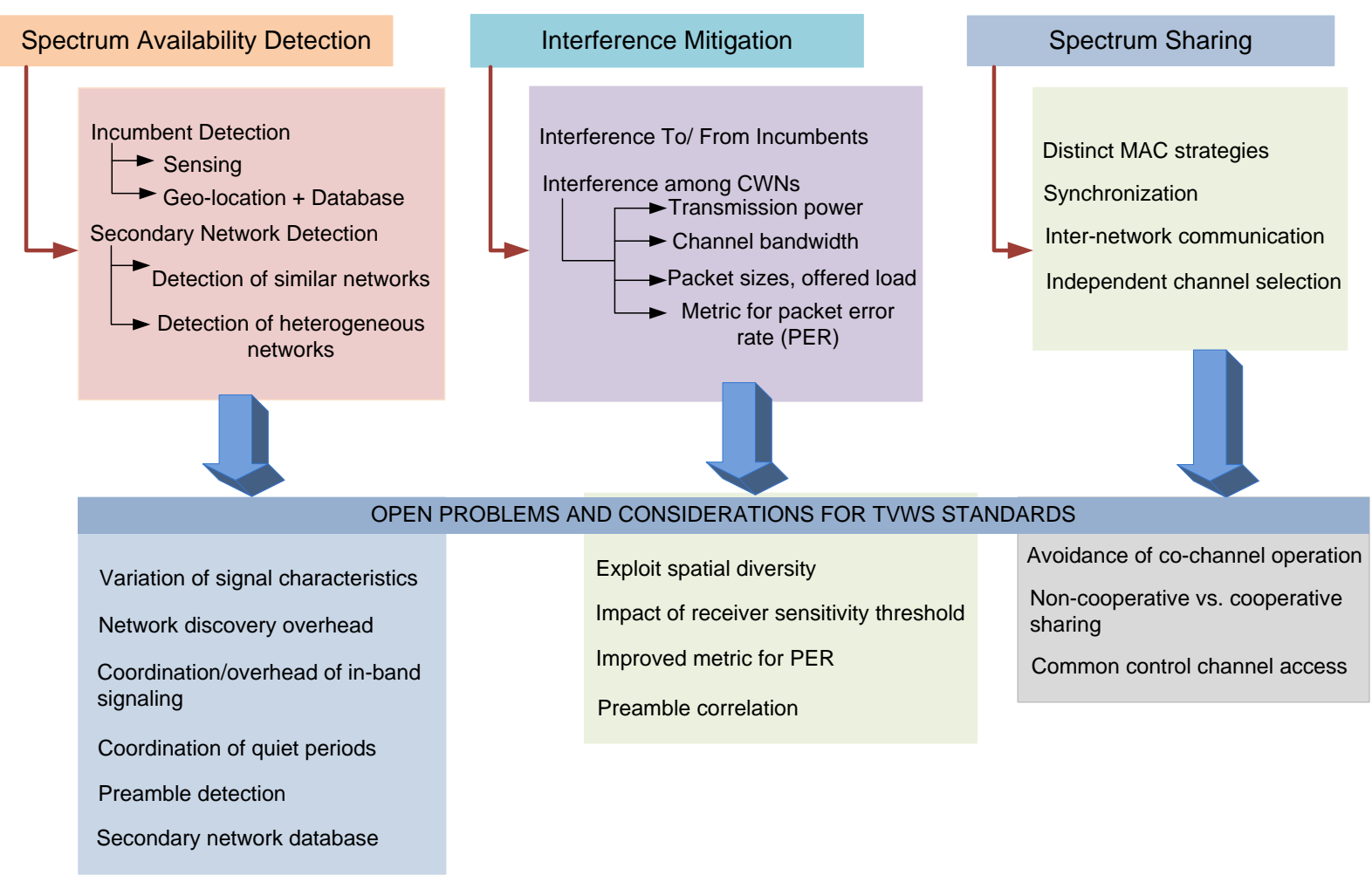

Fig. 2: Coexistence issues, open research problems, and considerations for TVWS standards enabling heterogeneous coexistence

incumbent users, their transmission power requirements, channels used, and expected duration of usage [6] . Secondary systems will query the WSD to determine availability of a TV channel while providing their own geographic locations. On receiving a query, the WSD sends information about the channels available at the specified location and allowed power levels for transmission [2] on such channels.

- Spectrum sensing: Process of scanning the RF spectrum in order to detect the presence of incumbent signals, usually above a certain sensing threshold, which defines the minimal signal level at which the incumbent signal must be detected. Any methodology used for spectrum sensing is calibrated in terms of two parameters namely, probability of false alarms $\left(P_{f a}\right)$ and probability of missed detections $\left(1-P_{d}\right)$. Typically there is a trade-off between sensing efficiency and the overhead required for sensing, i.e. sensing duration required to achieved a desired $\left(P_{d}\right.$, $\left.P_{f a}\right)$ Error! Reference source not found.

Spectrum sensing and WSD access will be key technologies to efficiently utilize the TVWS in most regulatory domains. Therefore, it is important to understand the potential detection challenges in heterogeneous scenarios.

\subsection{Incumbent detection challenges}

Geolocation and WSD access: As shown in Table I, certain secondary (fixed and Mode II) devices are required to self-geolocate in order to access the WSD. In fixed CWNs, the BS and CPEs will likely be equipped with satellite-based geolocation [6], although an alternative over-the-air 
mechanism is proposed in the 802.22 standard [3]. An over-the-air option could be used as backup in areas where satellite coverage is not available. In the case of Wi-Fi-like CWNs, access points (APs) will need to implement self-geolocation, in order to operate as master (Mode II) for lower power slave devices (Mode I). In many indoor use cases, availability of satellite signals could be an issue; hence over-the-air localization techniques and cooperation with other networks are feasible options, especially given the relatively low-resolution required by the geolocation mechanism (e.g. $+/-50 \mathrm{~m}$ proposed by the FCC).

Reliable spectrum sensing: Proposed techniques to date can be classified in five broad categories Error! Reference source not found.: (i) energy detection, (ii) waveform-based sensing, (iii) matched filtering, (iv) radio identification based sensing, and (v) cyclostationarity-based sensing. Energy detection does not require any prior information about the incumbent signal, but they do not perform well in cases where the detection threshold is very low, which is the case according to the TVWS requirements. Therefore, other techniques that use (varying) side information about the incumbent signals are more appropriate for TVWS, although performance and complexity varies Error! Reference source not found..

\subsection{Considerations for upcoming TVWS standards}

In order to increase sensing reliability, new standards for TVWS should introduce techniques to address the sensing coordination in heterogeneous scenarios. In 802.22, the BS schedules quiet periods for sensing during which no transmission takes place. Similar methods are used in Ecma 392 and may be introduced in 802.11af. Hence, coordination and synchronization of quiet times across CWNs is one possible option. Another approach could be to use sensing techniques that take into account the transmission characteristics of other CWNs in the sensing process for low $P_{f a}$. Furthermore, it is important to define not only standard sensing thresholds, but also minimal sensing requirements in terms of overhead needed to meet the regulations. The heterogeneous scenarios could also enable opportunities to share capabilities amongst networks. For instance, a Wi-Fi AP may be connected to the home CPE (Fig. 1) and share the satellite interface to obtain its own location and access the WSD through the 802.22 BS.

\section{Secondary user detection}

Future CWNs will also need to detect coexisting secondary systems operating in same or different TV channels. This will require detection of potentially different air interfaces. For instance, it will be critical for 802.11af and Ecma 392 networks to detect presence of nearby 802.22 networks since they will impose serious interference (see Section III-B) and avoid situations in which network capacity drops due to interference, while spectrum is not fully utilized. Now, we focus on detection challenges of similar and heterogeneous secondary networks.

\subsection{Detection of similar networks}

Coexistence of similar networks, also called self-coexistence, is considered in the scope of current standards, such as 802.22 [3]. The first step in any self-coexistence mechanism is the ability to detect neighboring networks. Otherwise, this may lead to following problems:

- Performance loss due to interference within the overlapping regions;

- Undetected asynchronous quiet periods among similar networks may result in transmission during sensing time and in turn, high $\mathrm{P}_{\mathrm{fa}}$; and

- Incomplete discovery of neighboring networks (i.e., hidden node problem) may cause data loss and impact the effectiveness of over-the-air (in-band) communication amongst networks. For example, the Coexistence Beacon Protocol (CBP) [3] packets exchanged 
between neighboring 802.22 BSs could be interfered by a third (hidden) network, preventing the self-coexistence process from converging.

The above problems highlight the importance of detecting similar networks, and some of the challenges include:

- Network discovery overhead: Most standards include some form of beacon transmission to facilitate network discovery. The 802.22 BS transmits regular Super-frame Control Header $(\mathrm{SCH})$ [3], which carry information about the cell and are transmitted using the most robust modulation/code option [2]. In the case of Ecma 392, all devices also transmit regular beacons Error! Reference source not found.. Similarly, 802.11af AP will also transmit regular beacons as the current 802.11 APs do. One fundamental difference in TVWS is the fact that the list of channels to be scanned may change dynamically and the frequency of scanning those channels may have to be increased due to the potential impact on incumbent protection (e.g. interference with incumbent sensing).

- Coordination and overhead of in-band signaling: The use of common control channels to enable network discovery has been proposed before for CWNs [6]. However the current and upcoming standards for TVWS are not expected to support a dedicated (out-of-band) over-theair control channel option. Instead, an in-band signaling approach has been adopted in 802.22 and Ecma 392 based networks. The CBP mechanism enables communication between 802.22 networks through a self-coexistence window (SCW) scheduled by BSs at the end of each frame [3]. However, it should be noted that SCWs incur a considerable overhead and should be used carefully. Notably, detecting such packets may require a relatively long scanning duration, adding to sensing overhead.

\subsection{Detection of heterogeneous CWNs}

Some of the main considerations include -

- Channel bandwidth definitions by each of the coexisting networks: Channelization bandwidths vary according to different networks. For example, 802.22 specifies $6 \mathrm{MHz}$ as operating bandwidth $^{3}$, while current $802.11 \mathrm{a} / \mathrm{b} / \mathrm{g}$ uses $20 \mathrm{MHz}$ bandwidth and upcoming 802.11af may use $5 \mathrm{MHz}$ or bonding of multiple channels up to $20 \mathrm{MHz}$.

- Transmission signal power variations among operating standards: Some networks have users with low power requirements while others have high power users. For example, 802.22 stations may transmit up to $4 \mathrm{~W}$ EIRP, while personal/portable devices under the current FCC rules are limited to a maximum of $100 \mathrm{~mW}$ EIRP. Detection of low power users will be a key issue.

- Signal characteristics among heterogeneous PHY modes: Broadcast DTV standard specifies known pilots and/or preambles, an inherent characteristic that is exploited for effective spectrum sensing. For secondary system signals, the available characteristics will differ from one standard to another and therefore need to be known in order to apply sensing based on signal characteristics. Otherwise, detection using signal characteristics is not a viable option.

\subsection{Considerations for upcoming TVWS standards}

Some of the possible solutions that could be adopted in upcoming standard to support efficient and reliable detection of CWNs in TVWS are:

\footnotetext{
37 or $8 \mathrm{MHz}$ channels may also be supported depending on the regulatory domain.
} 
- Intelligent management of out-of-band sensing: New standards should enable intelligent management of out-of-band ${ }^{4}$ sensing during stations' idle time together with cooperative sensing techniques. Furthermore, new standards will have to support reporting mechanisms which stations use to send spectrum utilization updates with respect to neighboring networks to a central spectrum manager or share with other peer stations in a distributed system.

- Preamble detection: Usually, a data packet consists of three sections namely, preamble, header, and data payload. Definition of a distinct preamble in a data packet for a CWN can help in the detection process. Correlation of received data packet preamble with known preamble sequences can be a potential solution to detection of heterogeneous networks. Lower values of correlation imply packets from undesirable networks and detection of the same.

- $\quad$ Secondary network database: A database approach for storing information about secondary systems may also help detection of fixed networks such as 802.22 , but it would be less effective for low-power personal/portable and peer-to-peer networks (e.g. 802.11af or Ecma 392 based) due to high mobility and need for connection to the infrastructure in order to update the database.

\section{B. Interference Mitigation}

Interference in the TVWS will be a challenging issue especially in areas of limited channel availability and where network coverage overlaps. Currently, heterogeneous networks share the unlicensed $2.4 \mathrm{GHz}$ band, and interference among them has been subject of extensive research [8]. Similar interference problems will exist as these technologies migrate into the TVWS. However, new interference situations will evolve in the TVWS, such as that between low power personal/portable devices (e.g. 802.11af and Ecma 392) and higher power fixed systems (e.g. 802.22). Furthermore, the good propagation characteristic of TVWS may also contribute to increased interference as transmission and interference ranges increase. For instance, Wi-Fi home networks typically operating co-channel without serious performance degradation in the $2.4 \mathrm{GHz}$ due to spatial reuse, could potentially experience more interference while operating in the same TV channel due to larger transmission and interference ranges. Last, but not least, interference from incumbents, mainly high power TV stations, is another specific problem to the TVWS. In summary, new interference scenarios specific to TVWS need to be addressed in the upcoming TVWS standards.

\section{Interference issues in heterogeneous $C W N$ scenarios}

Interference related issues in TVWs are classified under two categories: interference to/from incumbents and interference among CWNs.

\subsection{Interference to/from incumbents}

In addition to incumbent detection (discussed in Section III.A.1), requirements to limit out-of-band emissions are defined for all secondary devices, with extra restrictions on adjacent channel operation in order to reduce probability of interference on incumbents (Table I). On the other hand, high power incumbents (TV stations transmitting from 20 to $1000 \mathrm{KW}$ ) may also interfere with secondary systems. In some cases, these high power interferers may actually prevent secondary devices to report incumbent detection. Avoidance of such interference depends largely on location,

\footnotetext{
${ }^{4}$ Out-of-band refers to channels that are not the current operating channel $(\mathrm{N})$ or its first adjacent channels $(\mathrm{N}+/-1)$
} 
channel gain between the TV station and secondary users, and the difference in operating frequency between them. Location proximity as well as smaller differences (i.e., adjacent channels $\mathrm{N}+/-1$ of an active broadcasting channel $\mathrm{N}$ ) in their operating frequencies will severely degrade performance of a secondary network, which must include mechanisms to recover from such situations. One example is the incumbent detection recovery protocol adopted in the 802.22 standard that enable CPEs affected by strong incumbent interference to re-connect with its BS [3].

\subsection{Interference among $C W N s$}

Multiple CWNs may select the same TV channel due to uncoordinated selection process or limited availability. In such situations, ignorance of each other's transmission may result in overlapping packets. Consider the worst case situation in which all networks operate co-channel. Several interference problems could occur in such a set up, as shown in Fig 3. The main aspects that contribute to interference in TVWS scenarios are:

(1) Different Transmission powers: Transmit power control may result in better packet reception in the desired network but adversely affect other co-located networks. In addition, CPEs upstream transmissions may also contribute to interference to nearby networks that overlap with the main-lobe of the CPE's directional antenna. Since the CPE's back-lobe antennas gain is expected to be small, the CPEs interference range in other directions will be much smaller. For instance, mobile user 3 and the peer-to-peer network in Fig 3 will suffer stronger interference from CPEs 4 and 2, respectively, than the other secondary devices in the figure. Also, if CPE 4 in Fig. 3, away from the BS, increases its signal power for better performance, it may increase interference to neighboring co-channel users.

(2) Channel bandwidth: As illustrated before, 802.22 WRANs will operate on $6 \mathrm{MHz}$ wide channels while 802.11af standard may consider signal bandwidth of 5, 10, and $20 \mathrm{MHz}$ [9] based on availability of two or more contiguous channels. Suppose AP 2 in Fig. 3 operates using 4 contiguous TV channels using a $20 \mathrm{MHz}$ channel bandwidth mode. Hence, interference from the 802.22 BS and CPE 4, could affect only a section of the data packet received by the mobile user 3 under AP 2 in the WLAN.

(3) Offered load and packet size: The overlap in time between transmissions of heterogeneous CWNs will also result in interference and the degree of this overlap and the overall traffic load define the level of interference. Transmission time is directly proportional to packet size for a given data rate. It is intuitive that shorter packets incur lesser interference (i.e. lower packet loss probability) than larger ones, other things being equal. Additionally, packet loss due to interference is proportional to the offered load in the system.

(4) Inadequacy of Signal-to-Interference Ratio (SIR): Typically, SIR (or SINR more generally) is used as a surrogate for predicting packet error rate (PER). As is well-known, this implies treating interference as additive Gaussian noise; in heterogeneous scenarios, this is often insufficient Error! Reference source not found. and leads to incorrect estimates of PER which depends not only the interference power but on other aspects such as the modulation and coding.

\section{Interference related considerations for upcoming TVWS standards}

To combat the interference challenges that may evolve over time due to coexistence in the TVWS, we provide some considerations for upcoming TVWS standards: 


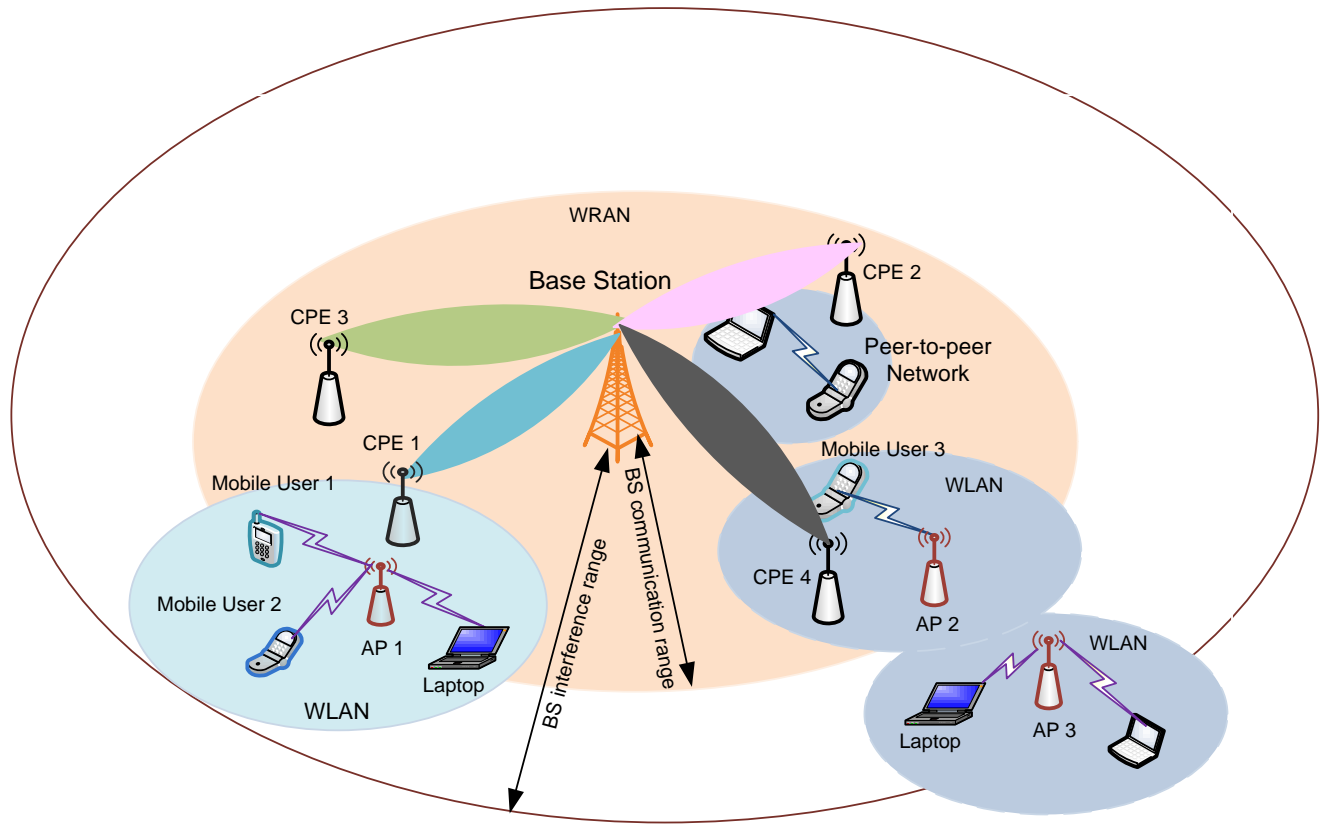

Fig. 3: Interference scenarios among heterogeneous cognitive wireless networks

Cooperative approaches can be utilized in terms of synchronization of quiet periods, sharing of sensing information as well as usage patterns between networks. In practice, however, implementing cooperation among competing networks is not a simple problem, as we discuss in the next section. Spatial diversity, in terms of multiple-input-multiple-output (MIMO) options can also be exploited with smart antenna technology to avoid interference from the direction of the interferer. For example, with spatial diversity embedded in the 802.22 and WLAN will mitigate interference in several scenarios depicted in Fig. 3. Directional antennas used by CPE 3 and CPE 1 will reduce interference on AP 1 and its associated stations.

Receiver sensitivity threshold: This threshold needs to be considered carefully in order not to trigger the receiver on unintended signal transmissions. This implies the idea of differentiating between users in the same network and the presence of interference from different networks. The thresholds can be set based on interference patterns of coexisting WRAN, WLAN, or WMAN networks.

?? Metric for PER analysis: Since SIR does not adequately capture the effects of modulation and coding, symbol-error rate can be used for better modeling of interference in the PHY layer and corresponding packet error rate analysis in scenarios of heterogeneous coexistence of secondary networks. Additionally, cyclic redundancy check for header and data payload sections of the received data packet need to be considered for precise interference analysis used for PER computations.

\section{Spectrum Sharing}

Avoiding operating channel overlap between CWNs is always desirable. However, given the dynamism of TVWS, it is possible that overlapping CWNs share available TVWS channels. Typical spectrum sharing solutions can be broadly classified as cooperative or non-cooperative 
mechanisms [10]. Examples of non-cooperative mechanism include power control and listen before talk features, such as CSMA/CA used in 802.11 networks. Cooperative schemes require coordination amongst coexisting networks. In the case of similar networks, implementation of both cooperative and non-cooperative approaches is facilitated by the fact that the networks operate according to the same PHY/MAC protocols. For instance, if all stations apply the same CSMA algorithm, some level of long term fairness can be achieved. Similarly, inter-network communication capabilities necessary for cooperative mechanisms is supported in 802.22. In heterogeneous CWNs, the spectrum sharing becomes even more challenging, given the intrinsic differences in the protocol stacks. The major spectrum sharing challenges in heterogeneous scenarios in TVWS are described next.

\section{Spectrum sharing challenges in heterogeneous $C W N S$}

Distinct MAC strategies: CWNs may operate according to different MAC techniques like time division multiple access (TDMA), frequency division multiple access (FDMA), code division multiple access (CDMA) or contention-based protocols. For instance, the 802.22 MAC is TDMAbased with PHY resources allocated on demand using OFDMA, while 802.11af will use its CSMAbased protocol and Ecma 392 uses a combination of reservation and contention based access. While 802.11af users could back-off when the medium is occupied by 802.22 transmissions, the other way around may not be true, since 802.22 devices do not need to listen before transmitting. The differences in MAC strategies may limit the effectiveness of non-cooperative list-before talk mechanism in achieving fairness in TVWS coexistence situations.

Inter-network communication: Currently, most MAC/PHY standards do not support over-the-air communication across heterogeneous networks, limiting the applicability of cooperative sharing strategies. For example, in order to achieve cooperative sharing mechanism between 802.22 BS and WLAN APs, these networks would have to negotiate usage of the same TV channel. One possible way is to multiplex transmissions of multiple overlapping networks in the time domain, as is done across 802.22 networks [3]. This sharing approach is illustrated in Fig. 4 where certain time slots are reserved for use by the 802.22 system, and others are reserved for contention-based access (WiFi clients). Although this concept seems simple, its implementation in the TVWS is not straight forward. First, it would require communication and negotiation between many competing networks. The 802.22 BS would cover a large number of 802.11 WLANs, Ecma 392 networks or other low power systems involved in the negotiation process. Second, the overhead in adapting the sharing schedule could be large depending on the number of coexisting systems and could also result in instability or convergence issues.

Synchronization: Assuming there are mechanisms that support the negotiation between heterogeneous CWNs, the implementation of such cooperative strategy would only be possible with tight time synchronization across all devices from different networks, which is a challenging problem. Although it is possible to keep tight synchronization within an 802.22 WRAN, or even across different WRANs, extending the synchronization to a potentially large number of personal/portable networks may not be possible, unless all systems and protocols are based on a universal reference clock.

Independent channel selection: Consider the scenario where one CWN is using TV channel A and having channel B as backup. Simultaneously, a second CWN operating in channel C (with channels $\mathrm{B}$ and D as backup options) detects the first network in channel A. Suppose the second network detects an incumbent on its operating channel. In the sequel, which backup channel should the second network move to (B or D)? Typically, channel selection is an implementation dependent 
procedure in most wireless standards. In TVWS, however, channel selection may be needed in more instances than just at network initialization, for example, to protect incumbents or to avoid co-

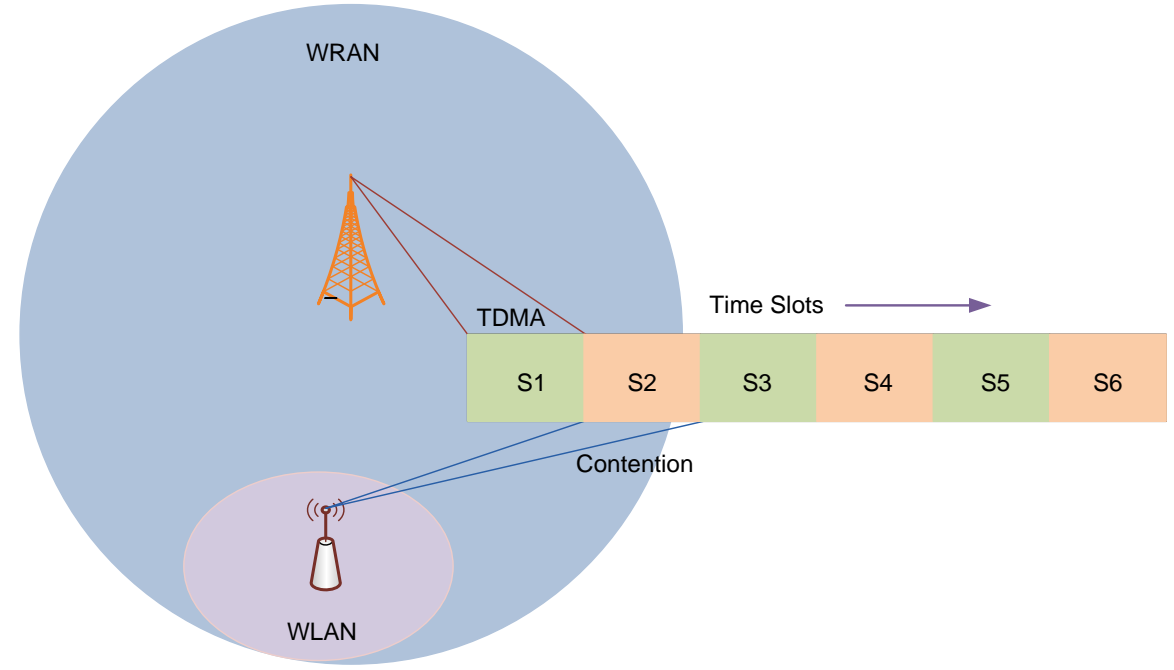

Fig. 4: Spectrum sharing between WRAN and WLAN in the TVWS

channel operation with other secondary networks, as in the example above. In this situation, completely independent selection procedure may results in sub-optimal operation. Both networks could end up in channel B after the incumbent is detected. Making use of sensing information about other secondary networks could be valuable in this example to avoid a co-channel operation.

\section{Spectrum sharing considerations in upcoming TVWS standards}

The first step towards efficient utilization of TVWS is to avoid co-channel operation, if enough channels are available. This can only be done with reliable network discovery mechanisms for heterogeneous scenarios (Section II.A). Furthermore, being able to detect specific characteristics (e.g. transmit power) or operational parameter, such as priority list of backup channels, of heterogeneous CWNs would also be useful to non-cooperative channel selection strategies that avoid co-channel operation. One example of such strategies is the spectrum etiquette mechanism adopted in the 802.22 standard to ensure neighboring WRANs reduce the probability of co-channel operation by selecting operation and backup channels that are less likely to be used by neighboring networks. This is achieve by exchanging information about backup channel lists, which would off course require some form of inter-network communications across heterogeneous CWNs. Another example of non-cooperative strategies for low power personal/portable devices is to give priority to the first adjacent channels of an active TV channel, since higher power fixed devices (e.g. 802.22 BSs and CPEs) are not allowed on adjacent channels according to the FCC rules. In this case, the personal/portable devices would still have to reduce the maximum power $(40 \mathrm{~mW})$, but this could be a good trade-off to avoid potential interference from high power secondary users in the area.

In case co-channel operation cannot be avoided, non-cooperative mechanisms to avoid interference could also be applied, but the effectiveness will depend on the characteristics of specific scenario including relative location of the devices, traffic load, transmit power, etc. The cooperative strategies that require inter-network communication and time synchronization are the most challenging as they would require a broad standardization effort across all secondary systems. There have been some proposals for utilizing a simple common control channel across networks in the context of the 802.19 coexistence standard, but it adds extra-cost and it is unclear whether other standards will reach a consensus on the "universal" PHY mode as the coexistence control channel. 
As discussed above, even if such control channel is available, the synchronization and negotiation process amongst competing secondary systems would still need to be addressed.

\section{CONCLUSION}

TVWS is considered for potential coexistence of heterogeneous CWNs including licensed, typically primary users, and unlicensed systems. This article briefly introduced the existing and upcoming standards in the TVWS and identified the prominent challenges to be encountered by these heterogeneous CWNs, while also taking into account the imposed regulations. Additionally, this article also provided insights and important considerations for the successful development of new wireless standards to achieve heterogeneous coexistence in the TVWS with an ultimate motif of efficient and enhanced spectrum utilization.

\section{References}

[1] J. Mitola, Cognitive Radio: An Integrated Agent Architecture for software defined radio. $\mathrm{Ph} . \mathrm{D}$. Thesis, Swedish, Royal Institute of Technology, 2000.

[2] Federal Communications Commission (FCC) 08-260: Second Report and Order and Memorandum Opinion and Order in ET Docket Nos. -2-380 (Additional Spectrum for unilicensed devices below 900 $\mathrm{MHz}$ and in the $3 \mathrm{GHz}$ band) and 04-186 (unlicensed operation in the TV broadcast bands), Nov. 14, 2008.

[3] IEEE 802.22 draft standard, “IEEE P802.22TM/D2.0 Draft Standard for Wireless Regional Area Networks," http://www.ieee802.org/22/, May 2009.

[4] J. Wang, M. Song,S. Santhiveeran, K. Lim,G. Ko, H. Kihong, S. Hwang, M. Ghosh, V. Gaddam, and K. Challapali, "First Cognitive Radio Networking Standard for Personal/Portable Devices in TV White Spaces," 2010 IEEE Symposium on New Frontiers in Dynamic Spectrum (DySpan), vol., no., pp.1-12, 69 April 2010.

[5] F. Granelli, at al "Standardization and Reseach in Cognitive and Dynamic Spectrum Access Networks: IEEE SCC 41 Efforts and Other Activities," IEEE Communications Magazine, January 2010.

[6] D. Gurney, G. Buchwald, L. Ecklund, S. Kufner, and J. Grosspietsch, "Geo-location database techniques for incumbent protection in TV White Space," 3rd IEEE International Symposium on New Frontiers in Dynamic Spectrum Access Networks, DySpan, 2008, Chicago, USA, Oct. 2008, pp. 1-9.

[7] T. Yucek and H. Arslan, "A survey of spectrum sensing algorithms for cognitive radio applications," IEEE Communications Surveys \& Tutorials, Volume 11, Issue 1, First Quarter 2009 Page(s):116 - 130.

[8] T. Henderson, G. Pei, R. Groves, T. Bosaw, M. Rush, C. Ghosh, and S. Roy, "Wireless Network Coexistence, "Boeing Project Report, Dec. 17, 2009.

[9] P. Bahl, R. Chandra, T. Moscibroda, R. Murty, and M. Welsh, "White space networking with Wi-Fi like connectivity," SIGCOMM 2009, Barcelona, Spain, pp. 27-38.

[10] P. Si, H. Ji, F. R. Yu, and V. Leung, "Optimal cooperative internetwork spectrum sharing for cognitive radio systems with spectrum pooling," IEEE Tran. On Vehicular Technology, Vol. PP, Issue 99, 2010, pp. $1-10$ 